\title{
Rôles exercés par le Niveau intermédiaire du système sanitaire en République démocratique du Congo : représentations des acteurs
}

\author{
Stakeholder representations of the role of the intermediate level \\ of the DRC health system
}

Jean Bosco Kahindo Mbeva ${ }^{1}$, Hermès Karemere ${ }^{2}$, Carole Schirvel $^{3}$, Denis Porignon ${ }^{4}$

\section{$\rightleftarrows$ Résumé}

Introduction : En République Démocratique du Congo (RDC), les structures intermédiaires du système de santé ont été pensées au cours des processus de mise en œuvre des soins de santé primaires dans une optique de soutien au district sanitaire. L'objectif de cette étude était d'explorer les représentations par les acteurs sanitaires du rôle exercé par ce Niveau intermédiaire (provincial).

Méthodes : Étude de cas unique basée sur l'analyse inductive des données d'interviews auprès de 27 informateurs-clés ayant œuvré au sein du système sanitaire de la RDC.

Résultats: Les représentations des acteurs ont révélé une évolution temporelle du rôle joué. Dénué de compétences et de financements suffisants, durant les années 80, le Niveau intermédiaire s'était davantage adonné à des fonctions d'inspection et de contrôle, rendant des comptes plus spécifiquement au niveau central du ministère de la Santé. Depuis la décennie 90, face à la demande pressante de soutien des équipes de district, dont l'autonomie de gestion a été mise à rude épreuve suite (i) aux urgences humanitaires, (ii) à la nécessité d'intégrer les programmes verticaux et (iii) aux logiques de nombreux acteurs, le Niveau intermédiaire du système sanitaire a développé des méthodes et des outils pour soutenir le district sanitaire. Ainsi a émergé un modèle subsidiaire du Niveau intermédiaire, dont l'efficacité est perçue variable selon les provinces ces dernières années.

Conclusion : D’après les représentations des acteurs et dans un contexte de décentralisation, le modèle «subsidiaire » du Niveau intermédiaire semble une alternative crédible au modèle « contrôle » en RDC.

Mots-clés: Connaissances, attitudes et pratiques en santé ; Personnel de santé ; Prestations des soins de santé ; République démocratique du Congo.

\section{$\rightleftarrows$ Summary}

Introduction: Intermediate health care structures in the DRC were designed during the setting-up of primary health care in a perspective of health district support. This study was designed to describe stakeholder representations of the intermediate level of the DRC health system during the first 30 years of the primary health care system.

Methods: This case study was based on inductive analysis of data from 27 key informant interviews..

Results: The intermediate level of the health system, lacking sufficient expertise and funding during the 1980s, was confined to inspection and control functions, answering to the central level of the Ministry of health and provincial authorities. Since the 1990s, faced with the pressing demand for support from health district teams, whose self-management had to deal with humanitarian emergencies, the need to integrate vertical programmes, and cope with the logistics of many different actors, the intermediate heath system developed methods and tools to support heath districts. This resulted in a subsidiary model of the intermediate level, the perceived efficacy of which varies according to the province over recent years.

Conclusion: The "subsidiary" model of the intermediary health system level seems a good alternative to the "control" model in DRC.

Keywords: Health knowledge, attitudes, practices; Health personnel; Delivery of health care; Democratic Republic of the Congo.

\footnotetext{
${ }^{1}$ Centre scientifique et médical - Université Libre de Bruxelles (CEMUBAC) - Route de Lennik, 808 - CP 595 - B-1070 Bruxelles - Belgique.

${ }^{2}$ Consultant indépendant - Canada.

${ }^{3}$ Fédération Wallonie Bruxelles - Direction de la surveillance et de la protection de la santé - Cellule de surveillance des maladies infectieuses

- Belgique.

${ }^{4}$ Université de Liège (ULG) - Belgique.
} 


\section{Introduction}

Au Nord comme au Sud, la décentralisation des systèmes de santé a conduit à la mise en place de structures provinciales publiques de gestion du secteur de la santé [1, 2, 3]. La préoccupation sous-jacente d'adéquation entre les politiques de santé et les attentes des populations vise des objectifs de qualité des services, d'efficacité, d'équité et d'efficience des systèmes de santé [2]. Cette préoccupation rejoint celles ayant motivé en 1988 l'attribution au niveau intermédiaire des fonctions de planification, de gestion des ressources, de coordination de l'action sanitaire, de gestion du personnel et de soutien, notamment logistique, au district de santé dans une perspective de renforcement de la mise en œuvre du district de santé [4]. En région africaine, l'option pour des systèmes de santé à trois niveaux, dont ce niveau intermédiaire (provincial), a été prise en 1985 lors du $35^{\mathrm{e}}$ sommet africain de l'OMS [5].

Plus spécifiquement, après son adhésion aux soins de santé primaires au début des années 80, la République Démocratique du Congo (RDC) a opté en 1987 pour un renforcement de ce niveau intermédiaire, faisant ainsi face aux difficultés rencontrées au cours des processus de développement du district de santé [6].

Une étude menée sous la direction d'Anne Mills et al. en 1991 dans certains pays européens, africains, asiatiques et américains, a contribué à mettre en évidence le rôle habituellement attribué au niveau intermédiaire [3], rôle qui rejoint globalement les fonctions prescrites en 1988. En dépit des résultats de cette étude, le niveau intermédiaire du système sanitaire n'a pas fait l'objet d'une attention suffisante dans les recherches menées sur les systèmes de santé basés sur le district de santé. De manière particulière, les études sur les représentations par les acteurs du rôle exercé par cet échelon en région africaine sont quasi inexistantes. L'attention a été davantage focalisée sur le district de santé. Entre le rôle attribué, ou plutôt prescrit [4], et le rôle réellement exercé, il pourrait être observé des écarts, susceptibles d'influer sur la qualité du soutien apporté au district de santé. La réalité de ces écarts et des facteurs auxquels ceux-ci seraient attribués peuvent être approchés au travers les représentations des acteurs œuvrant au sein ou en collaboration avec le Niveau intermédiaire du système sanitaire.

L'objectif de cette étude est d'explorer les représentations par les acteurs du rôle exercé par le Niveau intermédiaire du système sanitaire en RDC. Nous formulons au départ la proposition d'un changement temporel et spatial de modèle de Niveau intermédiaire en œuvre en RDC.

\section{Méthodes}

La méthodologie a été basée sur une étude de cas unique $[7,8]$ dans le cadre d'une étude globale sur les soins de santé primaires en RDC. Pour cette partie de l'étude, il s'agissait d'explorer la manière dont le Niveau intermédiaire s'est acquitté de ses responsabilités vis-à-vis des districts de santé, au travers l'analyse des représentations des acteurs.

\section{Cadre d'analyse}

Le modèle de la performance organisationnelle des organismes publics élaboré par Sicotte et al. [9] a été utilisé afin de nous doter d'un cadre d'analyse des représentations des acteurs. Ce modèle considère la performance comme une résultante d'un équilibre dynamique entre quatre fonctions principales : (i) le maintien des valeurs et d'un bon climat organisationnel, (ii) l'adaptation à un environnement changeant, (iii) la production des services, enfin (iv) l'atteinte des buts. Dans notre cadre d'analyse, les valeurs qu'il nous avait semblé utile de maintenir au Niveau intermédiaire sont celles prônées par les soins de santé primaires, à savoir la justice sociale, l'équité, la solidarité et la participation des personnes et des communautés aux actions visant l'amélioration de leur santé [10]. Le bon climat organisationnel était celui qui permet une action collective efficace et un épanouissement des personnels au sein de l'institution. Les services à produire étaient ceux qui permettent de répondre adéquatement aux attentes des parties prenantes, en particulier aux populations, en interagissant notamment avec les équipes des districts. Les buts à atteindre concernaient l'impulsion du développement et des performances du district de santé en termes de prise en charge sanitaire des populations.

\section{Collecte et analyse des données}

Cette étude est basée sur les résultats d'analyse inductive de données d'entretiens individuels. Les critères d'inclusion des informateurs-clés étaient d'avoir travaillé pendant au minimum 4 ans au sein du Niveau intermédiaire, ou en collaboration avec et dans au moins deux provinces de la RDC. La saturation en termes d'informations a été obtenue après des entretiens semi-structurés réalisés auprès de 27 acteurs, entre janvier et septembre 2010. Vingt des 
Tableau I : Dernière position occupée par les informateurs-clés interviewés dans le système sanitaire

\begin{tabular}{|c|c|c|c|c|}
\hline Partenaire/Ministère & Type de responsabilité & $\begin{array}{l}\text { Niveaux } \\
\text { du système sanitaire }\end{array}$ & Nb sujets interviewés & Code \\
\hline \multirow{6}{*}{ Ministère de la santé } & \multirow{3}{*}{ Horizontale } & Central & 4 & $\mathrm{MHC}$ \\
\hline & & Provincial & 5 & MHP \\
\hline & & Opérationnel & 1 & $\mathrm{MHO}$ \\
\hline & \multirow{3}{*}{ Verticale } & Central & 5 & MVC \\
\hline & & Provincial & 0 & MVP \\
\hline & & Opérationnel & 0 & MVO \\
\hline \multirow{6}{*}{$\begin{array}{l}\text { Partenaire du Ministère } \\
\text { de la santé }\end{array}$} & \multirow{3}{*}{ Horizontale } & Central & 6 & $\mathrm{PHC}$ \\
\hline & & Provincial & 2 & $\mathrm{PHP}$ \\
\hline & & Opérationnel & 1 & $\mathrm{PHO}$ \\
\hline & \multirow{3}{*}{ Verticale } & Central & 3 & PVC \\
\hline & & Provincial & 0 & PVP \\
\hline & & Opérationnel & 0 & PVO \\
\hline
\end{tabular}

vingt-sept personnes interviewées avaient œuvré aux trois niveaux du système sanitaire et leurs parcours professionnels combinés couvrent la totalité des 11 provinces de la RDC. La dernière position occupée est détaillée dans le tableau I. Sur base d'un guide d'entretien, les interviews ont porté sur cinq thématiques dans une logique d'approfondissement : (i) les perceptions globales des apports du niveau intermédiaire dans la mise en œuvre des soins de santé primaires; (ii) les perceptions des actions réelles, des activités menées par ce niveau; (iii) les perceptions du support apporté et des relations de ce niveau avec les zones de santé ${ }^{\text {; }}$ (vi) les facteurs ayant influé sur cette relation; (v) les effets liés au support apporté au niveau des zones de santé. Pour les thématiques (ii) à (v), un approfondissement d'entretien a permis d'obtenir les données pour les provinces dans lesquelles chacun avait travaillé et sur les changements observés au cours du temps. Ainsi l'ensemble des 11 provinces et la période allant de 1978 à 2008 ont été couvertes. Les entretiens ont duré entre 35 et 100 minutes et ont été enregistrés après accord préalable de chaque personne interviewée. Chaque entretien a été retranscrit par écrit. Les transcrits (109 pages pour l'ensemble) ainsi que les résumés ont été envoyés individuellement $(2$ à 4 pages) à chaque personne interviewée pour validation. Le résumé global des entretiens a été soumis pour enrichissement aux 27 personnes interviewées. En lien avec les objectifs de l'étude et en vue de donner du sens aux données

${ }^{1}$ En RDC, la zone de santé correspond au district de santé au sens de l'OMS. brutes, une analyse inductive des données d'interviews [11] a été réalisée. Selon cette démarche inductive, le codage des données et le développement des catégories à partir du verbatim a été réalisé. Ensuite, ces catégories ont été affinées à la suite de deux niveaux d'analyses par matrices : (i) l'analyse selon les quatre fonctions de performance d'après Sicotte [9], ensuite (ii) selon les provinces et le temps (matrice d'analyse temporelle et spatiale). Le codage et le dégagement des catégories ont été réalisés par le premier auteur, puis vérifiés par le deuxième auteur. L'anonymat sur la source des données d'interview a été observé pendant tout le processus de collecte et d'analyse des données.

\section{Résultats}

Les représentations des acteurs convergent sur la nécessité de disposer d'un Niveau intermédiaire du système sanitaire en RDC en vue d'adapter les politiques, les normes et les directives sanitaires aux particularités des zones de santé et d'en appuyer, au besoin en contrôler l'application dans un contexte d'un pays vaste et aux moyens de communication déficients. Un acteur l'illustrait comme suit : «c'est un très grand pays sans dispositif logistique efficace de communication [...] entre les provinces, les écosystèmes, les cultures, les dynamiques sociales et les profils de morbidité sont fort différents; les adaptations des politiques et des normes sanitaires sont donc très nécessaires [...] » (MHC). 
Perceptions du rôle exercé par Niveau intermédiaire du système sanitaire au cours des premières années de mise en œuvre des soins de santé primaires

Les premières années de mise en œuvre des soins de santé primaires remontent au début de la décennie 80. En rapport avec cette période, les acteurs interviewés ont mis en évidence un décalage des compétences et des ressources financières et matérielles entre les zones de santé et le Niveau intermédiaire, au détriment de ce dernier. Un ancien cadre du Niveau intermédiaire notait : « [...] moi, quand on m'a nommé médecin inspecteur provincial, je n'avais même pas de carrière derrière moi ; dans ces conditions que pouvaisje apporter de consistant aux zones de santé?» (MHC). Comme le note un ancien médecin chef de zone: "J'ai été médecin chef de zone, mais je pouvais faire toute une année sans une seule visite du Niveau intermédiaire» (MHP). Le Niveau intermédiaire a faiblement réalisé les activités de soutien au développement des zones de santé (renforcement des capacités du personnel, approvisionnement en médicaments essentiels...). Par contre, les actions les plus couramment réalisées ont été celles liées au contrôle et à l'inspection des structures sanitaires, y compris des laboratoires, des officines pharmaceutiques et des écoles de formation du personnel infirmier du niveau secondaire et de l'hygiène publique. Ces fonctions ont été exercées de manière prépondérante au cours des années 80 , dans une optique de mise en application stricte de la législation sanitaire et des directives nationales. Pour les activités liées à la surveillance épidémiologique et la lutte contre les épidémies, la préoccupation relevait de l'urgence de la préservation des vies menacées sur injonction implicite ou explicite de l'autorité provinciale. Un ancien médecin inspecteur précisait: " [...] on vous considère comme conseiller du Gouverneur; ce qui l'intéresse c'est que, s'il y a une catastrophe, il faut être capable d'intervenir » (PHC). Un partenaire ayant œuvré pendant plus de dix ans au niveau d'une zone de santé notait : « Pour moi, on voyait l'inspection provinciale de la santé (IPS) lors des épidémies ; il y avait des revues épidémiologiques, chaque lundi du mois et on en profitait pour parler d'autres problèmes de salaires, de médicaments [...]» (PHC). Pendant cette période, le Niveau intermédiaire a développé des liens plus rapprochés avec les autorités du Niveau provincial et national. Un autre ancien médecin inspecteur provincial notait : «En tant qu'autorité sanitaire provinciale, j'ai recherché la collaboration avec l'autorité politique, en lui faisant comprendre que ce que nous faisions était de son intérêt. Nous n'avions pas de ressources, il fallait renforcer plutôt les relations avec les partenaires opérationnels d'appui aux zones de santé» (PHC).
Pendant ce temps, le soutien au développement des soins de santé primaires a été délégué de manière tacite aux ONGs, aux églises et aux coopérations. Un ancien médecin chef de zone en donnait son interprétation : "[...] aux premières années des soins de santé primaires, je trouve que les coopérations, les églises et les ONGS nous ont beaucoup aidés dès le démarrage des zones de santé... les meilleures zones de santé, les meilleurs hôpitaux sont surtout ceux où ces partenaires se sont investis... là ils ont comme remplacé l'IPS dans le renforcement des capacités de prestations des soins et de gestion, l'approvisionnement en médicaments, le suivi... pendant ce temps, l'IPS comme hiérarchie sans ressources s'occupait plus de ses contrôles des établissements de soins, des pharmacies, de l'hygiène [...] (MHC)»

Ces représentations des acteurs sous-entendent la prédominance, pendant cette période, du «contrôle » dans l'exercice des fonctions du Niveau intermédiaire du système sanitaire, dans un contexte de pénurie de ressources à son niveau et de disponibilité de ressources au niveau des districts de santé.

\section{Un rôle de plus en plus accru du Niveau intermédiaire en direction des zones de santé depuis la fin de la décennie 90}

Contrairement à la période précédente, les années après la décennie 90 ont été caractérisées par la rupture de la coopération bilatérale, des crises sociopolitiques et le départ massif des compétences gestionnaires des zones de santé vers des postes plus rémunérateurs au sein d'ONGs et d'autres organisations en RDC et hors de la RDC. Cette situation a mis à rude épreuve l'autonomie de gestion des zones de santé. Depuis lors, une intervention plus engagée du Niveau intermédiaire a été sollicitée, apportée et mieux perçue par les équipes de gestion des districts de santé. Certains partenaires du secteur santé ont encouragé et soutenu cet appui. Un cadre de district de santé notait: "Cet accompagnement a été nécessaire car la gestion du district de santé était devenue et reste très complexe [...] faire de l'administration, prester des soins aux patients, communiquer avec la communauté, discuter avec les partenaires, jouer en même temps le rôle d'anthropologue, de sociologue, d'enseignant [...] avec les nombreuses crises humanitaires et le foisonnement des programmes verticaux, les demandes, les procédures et les outils des partenaires qui s'en occupent sont trop nombreux, souvent contradictoires et on doit faire avec tout ça au niveau de la zone de santé, ce qui n'est pas simple $[\ldots] »(\mathrm{MHO})$. 
L'appui de certains partenaires au Niveau intermédiaire a permis à ce dernier d'initier et développer des systèmes d'informations sanitaires au niveau des zones de santé. Des dispositifs d'approvisionnement en médicaments essentiels basés sur des centrales régionales de distribution des médicaments et des pharmacies centrales des zones ont été initiés et implémentés. Par rapport aux méthodes de travail, des espaces de dialogue entre cadres du Niveau intermédiaire, les partenaires et d'autres secteurs au Niveau provincial ayant une incidence sur la santé ont été initiés et développés. Un cadre du Niveau central notait: «le leadership de l'État a toujours posé problème, mais on a constaté que, malgré la crise, certaines provinces ont développé des espaces de concertation avec leurs partenaires avec un certain niveau de leadership [...]» (MHC). Ces espaces de dialogue ont par exemple permis d'adapter certaines stratégies initiées au Niveau central aux particularités des contextes des districts de santé. Ces espaces de dialogue ont également contribué à négocier la réallocation des ressources et l'adaptation des stratégies d'intervention en prenant en compte les avis des gestionnaires et les particularités des zones de santé. L'intérêt plus accru du Niveau intermédiaire à soutenir les zones de santé a mené à une pratique plus régulière des supervisions du personnel des zones de santé, souvent financées par les partenaires. Ce rôle a été perçu globalement pertinent pour certaines provinces. Un cadre du Niveau central du Ministère en donnait cette illustration : "[...] franchement je trouve que les choses ont beaucoup changé dans certaines provinces comme le Nord Kivu, Kinshasa et le Bas-Congo ces dernières années. J'ai plusieurs fois visité ces provinces dans le cadre de mes responsabilités actuelles. J'ai pu me rendre compte que les méthodes de travail ont beaucoup évolué, de nouveaux outils de travail ont été mis en place, les cadres de l'IPS se posent de bonnes questions sur leur propre travail, sur ce qu'ils apportent aux zones de santé [...] j'ai pu observer dans ces provinces que l'IPS renforce les capacités des cadres des zones de santé, approvisionne les zones de santé en vaccins, oriente les zones de santé vers les centrales d'approvisionnement en médicaments essentiels, mais n'interfère pas dans la gestion quotidienne de ces zones. Quand les équipes sont dépassées, par exemple lors d'une épidémie de choléra dans la zone de santé de Rutshuru, l'IPS intervient. Il y a quelques années quand nous étions encore au niveau des zones, le Médecin inspecteur contrôlait nos zones et pouvait décider de tout et à tout moment [...] c'était lui le chef!» (MHC).

Un cadre d'un programme spécialisé nuançait la portée des dynamiques pour certaines provinces : «[...] il est vrai on voit certaines bonnes initiatives dans certaines provinces [...] ce sont plus des provinces où l'IPS bénéficie d'un appui institutionnel de la part de l'une ou l'autre organisation partenaire du ministère de la santé [...] dans d'autres provinces par contre, les IPS sont sans moyens de leurs politiques malgré la bonne volonté et ça ne marche pas, les choses sont fragmentées» (MVC). Quelques invariants ont été relevés pour toutes les provinces : la coordination des financements est demeurée globalement déficiente et les hôpitaux provinciaux de référence sont restés en marge des processus de soutien aux zones de santé. Un cadre du Niveau central en donnait son illustration : "[...] des hôpitaux des zones de santé se sont mis à développer des pratiques au-delà de ce qui est acceptable; ils ont manqué de lieux de référence utiles des malades, des stages pour cliniciens, pour améliorer leurs pratiques cliniques» (MHC). Un cadre du niveau central observait : «on a vite compris que les inspections provinciales de la santé, dans la configuration actuelle, n'avaient pas une bonne architecture pour accompagner les zones de santé et qu'il fallait autre chose » (MHC). Le processus de renforcement du Niveau intermédiaire a néanmoins été confronté aux logiques de certains programmes verticaux. Un cadre du Niveau central notait : «Le médecin inspecteur provincial doit tout coordonner, mais lui-même et son équipe sont sans moyens suffisants ; dans l'entretemps, on a créé des coordinations provinciales des programmes, privant l'inspection du peu des ressources disponibles [...] » (MVC).

En dépit de ces faiblesses, les pratiques jugées positives ont été reprises dans des projets négociés par le Ministère pour appuyer le Niveau intermédiaire dans d'autres provinces et ont conduit à prévoir un axe de réorganisation du Niveau intermédiaire dans la première édition de stratégie de renforcement du système de santé en RDC (SRSS). La perception du Niveau intermédiaire, pour ces dernières années, met en exergue des fonctions plus orientées vers les cadres des districts de santé et les partenaires et visant le soutien aux zones de santé, en étant attentif à ses préoccupations et en renforçant progressivement l'autonomie de ses équipes. Ces changements ont fait émerger un nouveau modèle de Niveau intermédiaire, ayant le principe de subsidiarité au cœur de son action vis-à-vis des zones de santé ces dernières années, bien que variables selon les provinces et ayant laissé en marge les hôpitaux provinciaux de référence dans ce processus.

\section{Discussion}

Les perceptions du rôle joué par le Niveau intermédiaire du système sanitaire en RDC ont mis en évidence un important changement du modèle principal du Niveau 


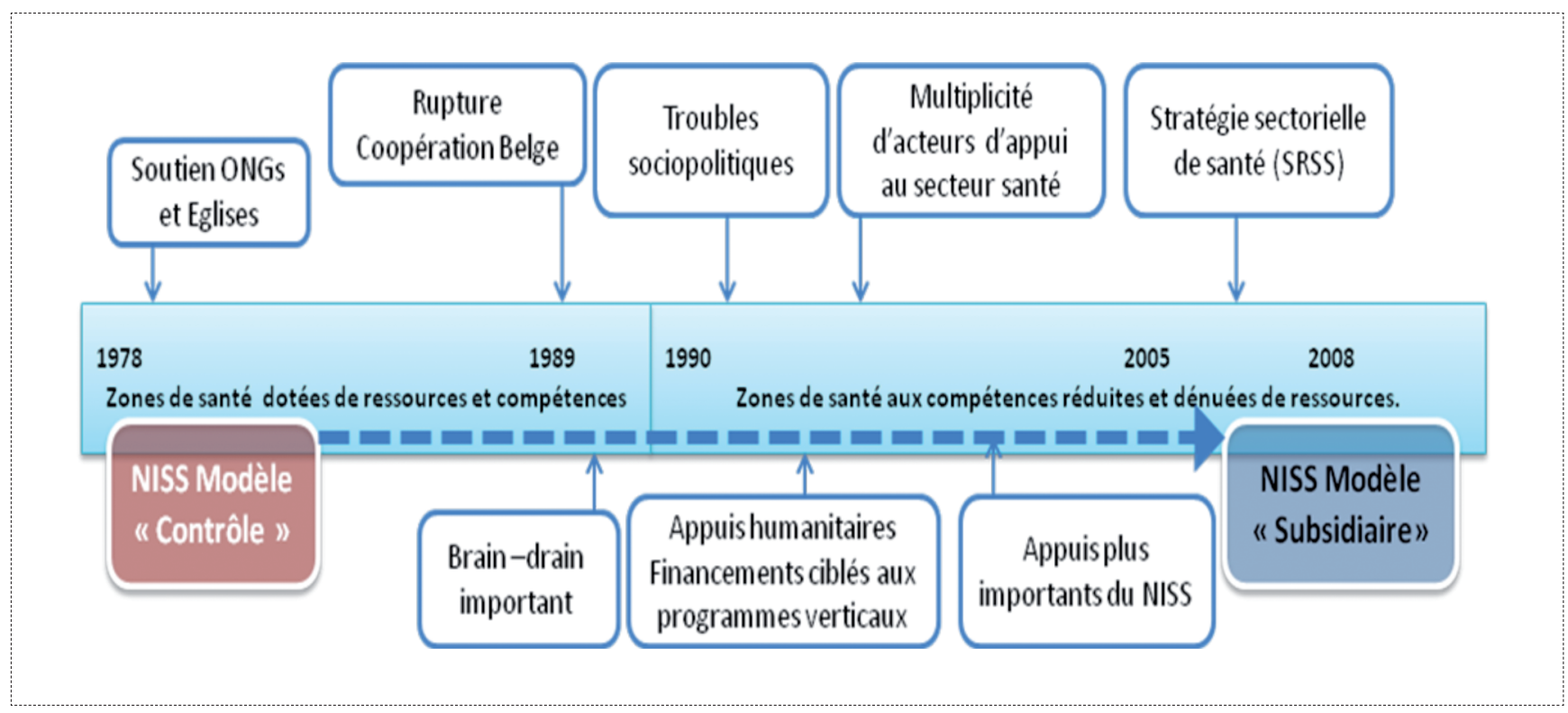

Figure 1 : Ligne du temps des facteurs ayant influé sur l'évolution du modèle

intermédiaire. Du modèle "contrôle », qui renvoie à la dénomination initiale du Niveau intermédiaire du système sanitaire en RDC («inspection provinciale de la santé »), il a été observé à des degrés variables selon les provinces, l'émergence d'un nouveau modèle «subsidiaire ». Le rôle exercé par le Niveau intermédiaire dans les provinces ces dernières années, renvoie plus soit à l'ancien soit au nouveau modèle. Ces résultats semblent conforter la proposition initiale de variation spatiale et temporelle de modèle de Niveau intermédiaire du système sanitaire en RDC.

Une variation spatiale des modèles de régionalisation des systèmes de santé a été décrite au Canada [12] et en Italie [13]. En Italie, cette variation est liée à des processus historiques de luttes sociales et de compromis des groupes d'intérêts institutionnels et non institutionnels.

Il importe à présent de discuter les déterminants et les caractéristiques de deux modèles théoriques du Niveau intermédiaire en RDC.

\section{Déterminants de deux modèles}

Un premier facteur qui paraît avoir déterminé la variation temporelle des fonctions exercées semble être le changement intervenu dans les attentes des districts de santé. Pendant la décennie 80, étant donné les bonnes compétences au niveau des zones de santé ayant rayonné hors de la RDC [14], le Niveau intermédiaire dont le gradient de compétences jouait plutôt en sa défaveur ne pouvait être sollicité. Cette situation expliquerait le fait que la fonction de soutien au développement des soins de santé primaires s'est peu développée pendant la décennie 80 (Figure 1).

En tant que structure déconcentrée du Niveau central, elle a servi d'outil de contrôle du respect de la législation et des directives nationales au niveau des provinces plus dans une logique de reddition des comptes envers les autorités centrales et provinciales. Les interventions lors des épidémies semblent aussi répondre à cette même logique. Ces éléments paraissent rejoindre l'affirmation de certains auteurs qui ne reconnaissent pas à la déconcentration les bénéfices d'une vraie décentralisation [5].

Par contre, bien que les prescrits de fonctions et la dénomination officielle du Niveau intermédiaire n'aient pas été modifiés au cours de la décennie 90, les changements du modèle semblent avoir été davantage induits par les demandes implicites et explicites des zones de santé. En effet, les difficultés rencontrées pour concilier les logiques de nombreux acteurs et répondre aux défis des urgences humanitaires et d'intégration des programmes spécialisés, ont suscité des demandes pressantes des zones de santé vis-à-vis du Niveau intermédiaire. La réponse à ces défis nécessitait les ressources et les compétences au Niveau intermédiaire. La variation des compétences et des financements selon les provinces pourrait, aussi et en partie, expliquer la variation selon les provinces de la qualité du soutien apporté aux districts de santé. La qualité des compétences au Niveau intermédiaire requiert néanmoins d'être explicitée. Elle pourrait être appréciée au travers de 


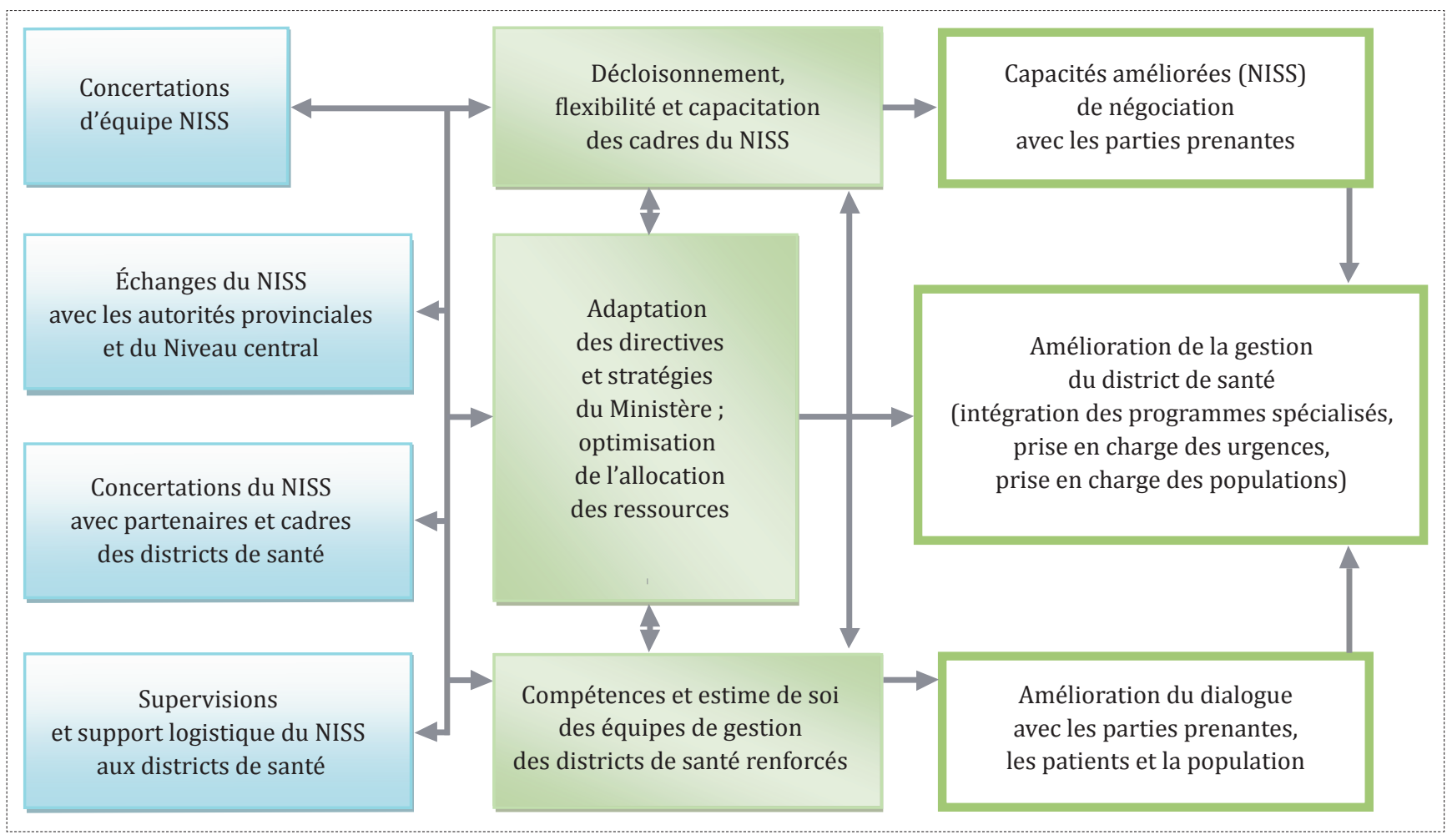

Figure 2 : Représentation des dynamiques du modèle " subsidiaire " du Niveau intermédiaire du système sanitaire

la qualité des interactions développées, en interne au sein des structures et en externe avec les principaux acteurs en présence. Pour les provinces dont les acteurs ont dynamisé les districts de santé, des modifications importantes du fonctionnement interne du Niveau intermédiaire et de ses interactions avec les autres acteurs, ont été observées dans certaines provinces comme le Nord Kivu et Kinshasa. D'où l'hypothèse d'une interaction entre la structure et les fonctions exercées au Niveau intermédiaire du système sanitaire.

\section{Caractéristiques de 2 modèles de Niveau intermédiaire}

Pour le modèle " contrôle », le respect des procédures et le respect de l'autorité établie, de la hiérarchie à qui la structure et son personnel sont redevables semblent être une caractéristique essentielle. Le soutien au processus de renforcement des compétences et de résolution des problèmes des zones de santé est facultatif et la sanction négative fréquente. Ce modèle contrôle semble s'accommoder à une bureaucratie, structure très peu flexible [15] et dont les difficultés d'alignement stratégique ne peuvent qu'entraver les fonctions de production et d'atteinte des buts décrites par Sicotte [9]. Ces caractéristiques pourraient expliquer l'inadéquation prolongée de la réponse du Niveau intermédiaire aux difficultés auxquelles ont été soumises les zones de santé de la RDC dès le début de la décennie 90 dans bien des provinces. Par contre, le modèle « subsidiaire » (Figure 2), intègre la suppléance et l'intervention dans sa relation avec le district de santé. Pour répondre à ces deux impératifs, le Niveau intermédiaire est plus à l'écoute des préoccupations des zones de santé et à celles des parties prenantes au développement des zones de santé. Ce qui renforce les fonctions d'adaptation et d'atteintes des buts proposées par Sicotte [9].

Avec le modèle "subsidiaire ", la qualité des interactions avec les différents acteurs est importante et conditionne la qualité du soutien au développement du district sanitaire. Les processus de développement du district sanitaire étant complexes, les dynamiques d'apprentissage au travers les interactions semblent être une autre caractéristique importante de ce modèle subsidiaire, qui renforce les fonctions d'adaptation et de maintien d'un climat organisationnel décrits par Sicotte [9]. Enfin, ce modèle ne pourrait s'accommoder à une structure bureaucratique. Par le dialogue engagé avec les partenaires financiers et les équipes des zones de santé, ce modèle permettrait de répondre aux objectifs d'efficience d'allocation des ressources mobilisés 
auprès des partenaires pour une meilleure prise en charge des populations ayant, en partie, motivé la décentralisation des systèmes de santé [16]. Certains auteurs affirment néanmoins qu'il n'existe pas de résultats scientifiques tangibles qui confirment avec certitude la supériorité des systèmes régionalisés en ce qui concernent leur efficience, leur efficacité et la qualité de la participation citoyenne [17].

Pour le cas de la RDC, le système de district de santé mis en œuvre a rayonné même en dehors de ses frontières [14]. Néanmoins, la réalisation de ces objectifs ne pourrait être attribuée au modèle contrôle du Niveau intermédiaire de l'époque, qui était plus au service du Niveau central et de l'autorité politique provinciale. Elle serait plus liée à la qualité des ressources humaines des zones de santé, qui a été soutenue par les partenaires d'appui aux districts sanitaires. Sans contester la responsabilité reconnue à l'État dans le pilotage du système sanitaire [18], les résultats de cette étude indiquent que la clé de la réussite se trouve dans la qualité des interactions entre les principaux acteurs en présence, dans un contexte donné, dans une perspective globale de renforcement du système sanitaire. Ainsi, le modèle subsidiaire favorise un équilibre dynamique entre les quatre fonctions du modèle développées par Sicotte, en vue d'une performance organisationnelle au Niveau intermédiaire, dont les effets sont d'impulser une bonne fonctionnalité du district de santé à répondre aux besoins de santé des populations. Le modèle «subsidiaire » incite à une gouvernance distribuée et adaptative entre différents acteurs au Niveau intermédiaire, qui se nourrit des interactions évoquées plus haut et du dialogue. Ces interactions supposent de la réflexivité, une disposition des équipes à désapprendre et à apprendre. Les structures du Niveau intermédiaire doivent se prêter à cette flexibilité. Ainsi, une troisième hypothèse de travail émerge : celle de la restructuration du Niveau intermédiaire en fonction de ses finalités, en prenant en compte les particularités du contexte de l'action.

\section{Limites de l'étude}

Les résultats de cette étude sont essentiellement basés sur des entretiens individuels. Bien que de nombreux informateurs œuvrent depuis la décennie 80 en RDC, le risque de biais de mémoire ne saurait être complètement écarté. Ainsi, des études mobilisant d'autres sources d'évidence, des données quantitatives et qualitatives et d'autres plus ciblées sur des provinces, sont requises pour approfondir les caractéristiques et les principes au cœur du modèle subsidiaire du Niveau intermédiaire.

\section{Conclusion}

L'émergence en RDC du modèle « subsidiaire » du Niveau intermédiaire est une alternative crédible au modèle «contrôle» en RDC. Ce nouveau modèle «subsidiaire» semble, au cours des dernières années, en adéquation avec le principe de dévolution exprimé par la constitution de la RDC promulguée en 2006 [19] et le principe de renforcement du système de santé porté par la stratégie sectorielle (SRSS) [20]. Tous deux prônent un rôle plus accru du Niveau intermédiaire du système sanitaire et du district de santé. Ce modèle est susceptible de suppléance et d'intervention qui renforce l'autonomie et l'estime de soi des équipes de gestion des districts de santé face à la complexité liée notamment aux urgences, à la multiplicité d'acteurs et à la nécessité d'intégrer les activités des programmes verticaux.

Aucun conflit d'intérêt déclaré

\section{Remerciements}

Les auteurs remercient les acteurs qui se sont prêtés aux interviews.

\section{Références}

1. Saltman RB, Bankauskaite V, Vrangbaek K. Decentralization in health care: strategies and outcomes. Londres: McGraw Hill - Open university press; 2007. 326 p. (European observatory on health systems and policies). [Visité le 06/08/2014]. En ligne: http:// www.euro.who.int/__data/assets/pdf_file/0004/98275/ E89891.pdf.

2. Bossert T. Analyzing the decentralization of health systems in developing countries: decision space, innovation and performance. Soc Sci Med 1998;47(10):1513-27.

3. Vaughan JP. Leçons de I'expérience. In: Mills A, Vaughan JP, Smith DL, Tabibzadeh I, éditeurs. Décentralisation des systèmes de santé. Concepts, problèmes et expériences de quelques pays. Genève : Organisation mondiale de la santé ; 1991. pp. 157-174. [Visité le 06/08/2014]. En ligne: http://apps.who.int/iris/ bitstream/10665/40744/2/9242561371_\%28p81-p174\%29. pdf?ua=1.

4. Organisation mondiale de la santé (OMS). La mise en œuvre : un véritable défi. Des systèmes de santé de district pour renforcer les soins de santé primaires. Genève : OMS; 1988. 74 p. [Visité le 06/08/2014]. En ligne: http://apps.who.int/iris/ bitstream/10665/58175/1/WHO_SHS_DHS_88.1_Rev.1_fre. pdf ?ua $=1$ 
5. Perrot J. La décentralisation administrative et la contractualisation. In : Perrot J, de Roodenbeke $\mathrm{E}$, édtieurs. La contractualisation dans les systèmes de santé. Paris : Karthala ; 2005. pp. 211-230.

6. Kalambay K, Fondation Hanns-Seidel. Charte de Mbanza Ngungu : une stratégie de collaboration intrasectorielle entre les partenaires du secteur santé au Zaire : actes de la conférence-atelier. Kinshasa : Département de la santé publique et des affaires sociales; 1987. $172 \mathrm{p}$.

7. Yin RK. Case study research: design and methods. $3^{\mathrm{e}}$ ed. Los Angeles (CA): Sage publications; 2003. 181 p.

8. Gagnon YC. L'étude de cas comme méthode de recherche : guide de réalisation. Québec (Canada) : Presses de l'Université du Québec; 2008. $128 \mathrm{p}$.

9. Sicotte C, Champagne F, Contandriopoulos D. Performance organisationnelle des organismes publics de santé. Ruptures, revue transdisciplinaire en santé. 1999;6(2):34-46.

10. Organisation mondiale de la santé (OMS). Déclaration d'Alma-Ata sur les soins de santé primaires [Page internet]. c2014. [Visité le 06/08/2014]. En ligne: http://www.who.int/topics/primary_ health_care/alma_ata_declaration/fr/.

11. Blais $\bar{M}$, Martineau $S$. L'analyse inductive générale: description d'une démarche visant à donner un sens à des données brutes. Recherches Qualitatives. 2006;26(2):1-18.

12. Denis JL, Contandriopoulos D, Langley A, Valette A. Les modèles théoriques et empiriques de régionalisation du système sociosanitaire. Montréal : Université de Montréal, Faculté de médecine, Groupe de recherche interdisciplinaire en santé (GRIS) ; 1998. 144 p.
[Visité le 06/08/2014]. En ligne : http://www.santecom.qc.ca/ BibliothequeVirtuelle/GRIS/2921954257.pdf.

13. Vicarelli G. Du centralisme napoléonien au fédéralisme administratif. Les processus de décentralisation en Italie. Revue Sociologie Santé. 2010;32:23-53.

14. Van Lerberghe W. Les politiques de santé africaines : continuités et ruptures. Bulletin des séances. Académie royale des sciences d'Outre-Mer. 1993;39(2):205-30.

15. Mintzberg H. Structure et dynamique des organisations. $9^{e}$ édiction). Paris : Éditions d'Organisations ; 2006. 434 p. (Les références).

16. Pavolini E. La décentralisation du système sanitaire fait-elle du bien à la santé ? Quelques réflexions à partir du cas italien. Revue Sociologie Santé. 2010;57-81.

17. Saltman RB. Decentralization, re-centralization and future European health policy. Eur J Public Health. 2008;18(2):104-6.

18. Organisation mondiale de la santé (OMS). Rapport sur la santé dans le monde, 2000 : pour un système de santé plus performant. Genève: OMS ; 2000. 248 p. [Visité le 06/08/2014]. En ligne: http://www.who.int/whr/2000/en/whr00_fr.pdf?ua=1.

19. République Démocratique du Congo, Assemblée nationale. Constitution de la République démocratique du Congo. Kinshasa: République démocratique du Congo; 2006. 57 p. [Visité le 06/08/2014]. En ligne : http://democratie.francophonie.org/ IMG/pdf/Constitution_de_la_RDC.pdf.

20. République Démocratique du Congo, Ministère de la santé publique, Secrétariat général. Stratégie de renforcement du système de santé en

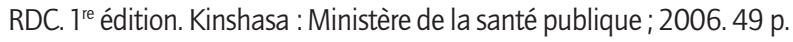


